\title{
Urologist
}

Cape Breton Regional Hospital and Nova Scotia Health Authority Sydney, NS, Canada

Opportunity Type: Permanent Fulltime

Estimated Annual Salary: Competitive Remuneration

Type of Remuneration: FFS - Fee for Service

NSHA Incentive: Site Visit \& Relocation Allowance Programs

Department of Health and Wellness Incentive: Debt Assistance, Tuition Relief

\section{About the Opportunity:}

The Department of Surgery at Cape Breton Regional Hospital seeks to recruit a full time Urologist, preferably with Canadian certification. The successful applicant will be part of a team of 3 Urologist serving the Cape Breton Regional Municipality and the surrounding communities with a catchment area of over 150,000.

Cape Breton Regional Hospital is a 250 bed tertiary care institution that provides a broad range of medical care to patients in the Eastern Zone of the Nova Scotia Health Authority. The Urology service at the Cape Breton Regional Hospital is equipped to provide a full range of specialised surgical care. The urology service provides ER on-call coverage 24/7 for the Cape Breton Regional Municipality and surrounding area.

\section{Qualifications:}

Candidates must be certified, or eligible for certification, by the Royal College of Physicians and Surgeons of Canada, eligible for licensure with the College of Physicians and Surgeons of Nova Scotia and satisfy the credentialing requirements of the Nova Scotia Health Authority.

\section{Community Details:}

Sydney is a vibrant port city on the edge of the beautiful Cape Breton Highlands National Park. Cape Breton Regional Municipality is the second largest municipality in the province of Nova Scotia. With a population of approximately 115,000, Cape Breton Island is known for its rich Celtic culture and stunning scenery. You will find an island of magnificent natural beauty yet very modern. We host an array of shops, restaurants, golf courses, entertainment and amenities including an airport and cruise ship terminal. In addition, the region remains young, diverse and vibrant as it is the home to Cape Breton University.

\section{Interested persons should contact:}

Bill O'Brien

Physician Recruitment Consultant

Nova Scotia Health Authority, Easter Zone

902-574-3189; Bill.obrien@nshealth.ca 